\title{
Measurement of Plasma Glucagon Levels Using Mass Spectrometry in Patients with Type 2 Diabetes on Maintenance Hemodialysis
}

\author{
Yuta Yoshizawa ${ }^{a}$ b Michihiro Hosojimac Hideyuki Kabasawac \\ Naohito Tanabe ${ }^{d}$ Atsushi Miyachi $^{\mathrm{e}}$ Hitoshi Hamajima $^{\mathrm{e}}$ Eri Mieno $^{\mathrm{e}}$ \\ Masaki Kobayashi $^{f}$ Tadahiro Kitamura $^{f}$ Ichiei Narita $^{b} \quad$ Akihiko Saito $^{a}$ \\ aDepartment of Applied Molecular Medicine, Kidney Research Center, Niigata University Graduate School of \\ Medical and Dental Sciences, Niigata, Japan; ${ }^{b}$ Division of Clinical Nephrology and Rheumatology, Kidney Research \\ Center, Niigata University Graduate School of Medical and Dental Sciences, Niigata, Japan; 'Department of Clinical \\ Nutrition Science, Kidney Research Center, Niigata University Graduate School of Medical and Dental Sciences, \\ Niigata, Japan; 'Department of Health and Nutrition, Faculty of Human Life Studies, University of Niigata Prefecture, \\ Niigata, Japan; 'Radioisotope and Chemical Analysis Center, Sanwa Kagaku Kenkyusho Co., Ltd., Mie, Japan; \\ ${ }_{\mathrm{f}}$ Metabolic Signal Research Center, Institute for Molecular and Cellular Regulation, Gunma University, Gunma, Japan
}

\section{Keywords}

Plasma glucagon - Liquid chromatography-high-resolution mass spectrometry $\cdot$ Hemodialysis

\begin{abstract}
Background: Recently, attention has been focused on the effect of glucagon on blood glucose variability. The dynamics of glucagon have attracted attention as a new target in the treatment of diabetes patients. However, the dynamics of glucagon in hemodialysis (HD) patients with type 2 diabetes mellitus (T2DM) remain unclear. Objectives: The aim of this study was to assess the dynamics of glucagon in HD patients with T2DM. Materials and Methods: We measured plasma glucagon in HD patients with T2DM by liquid chromatography-high-resolution mass spectrometry (LC-HRMS), sandwich enzyme-linked immunosorbent assay (ELISA), and radioimmunoassay (RIA). The glucagon levels measured by each method were compared. We used the glucagon levels determined by our developed LC-HRMS method as the standard in this study. Results: Plasma glucagon levels measured
\end{abstract}

karger@karger.com www.karger.com/kbr

Karger"

BOPEN ACCESS
(C) 2021 The Author(s)

Published by S. Karger AG, Basel

This is an Open Access article licensed under the Creative Commons Attribution-NonCommercial-4.0 International License (CC BY-NC) (http://www.karger.com/Services/OpenAccessLicense), applicable to the online version of the article only. Usage and distribution for commercial purposes requires written permission. by LC-HRMS before HD were significantly higher than those measured after HD. Plasma glucagon levels measured using sandwich ELISA had a significantly higher correlation with those measured using LC-HRMS compared with RIA. Conclusions: This was the first study to assess glucagon levels in $\mathrm{HD}$ patients with T2DM using LC-HRMS, which is considered a highly accurate method. Sandwich ELISA was shown to measure glucagon levels accurately as well.

○) 2021 The Author(s)
Published by S. Karger AG, Basel

\section{Introduction}

The dynamics of glucagon are receiving considerable attention as a new target in the treatment of diabetes [ 1 , 2]. Achieving good glycemic control is important, even in hemodialysis (HD) patients with type 2 diabetes (T2DM). However, there are differences in the dynamics of many hormones between HD patients and non-HD patients. Moreover, HD-induced hypoglycemia, which might be associated with the dynamics of these hormones, often 
occurs in HD patients, especially during HD sessions. Therefore, it is important to investigate the dynamics of hormones such as glucagon in HD patients, but the details are still not known. Recently, a quantitative method for measuring glucagon by liquid chromatography-highresolution mass spectrometry (LC-HRMS) was developed [3]. Although this method is time consuming, it is considered highly accurate [4]. However, no published reports have examined the plasma glucagon levels measured by LC-HRMS (G-LC/MS) in HD patients with T2DM. In particular, it has been reported that plasma glucagon levels measured by radioimmunoassay (G-RIA) decrease before and after a single HD session [5-7], but the details were not elucidated. Holst et al. [8] reported that accurate measurements of glucagon could be performed using a sandwich enzyme-linked immunosorbent assay (ELISA) approach. The accuracy of this method was also confirmed by comparing the results with those of LC-HRMS [1]. Currently, sandwich ELISA might be a useful option for measuring glucagon in patients with T2DM [9], but the accuracy of sandwich ELISA in measuring glucagon of HD patients with T2DM remains unclear. Accordingly, in this study, we utilized LC-HRMS, sandwich ELISA, and RIA to measure glucagon levels in HD patients with T2DM before and after a single HD session in order to cross-validate the use of these methods.

\section{Materials and Methods}

\section{Patients}

Plasma samples from HD patients with T2DM were obtained from a previously conducted clinical study (UMIN000024284). This study was approved by the institutional review boards of Niigata University (Approval No. 2015-2572) and Shinrakuen Hospital (Approval No. H28003). All procedures were performed in accordance with the Helsinki Declaration of 1964 and its later amendments and adhered to national regulations. This research was conceived as a retrospective study based on previously collected data, and all patients provided written informed consent for using their clinical data for research purposes.

\section{Sample Collection}

We evaluated 52 plasma samples (26 patients from 3 HD centers) for comparison of glucagon assays before and after a single HD session at the beginning of the clinical study. Samples were obtained for glucagon measurements in the middle of the week. The subjects rested on the bed and did not have meals during HD sessions.

Blood samples were collected using evacuated tubes (BD P800; Becton Dickinson, Tokyo, Japan). Plasma samples were separated by centrifugation for $20 \mathrm{~min}$ at $3,000 \mathrm{rpm}$ and $4^{\circ} \mathrm{C}$, dispensed into plastic tubes to avoid the occurrence of freeze-thaw cycles, and stored at $-20^{\circ} \mathrm{C}$ or below.

Glucagon Levels in Hemodialysis Patients

\section{Plasma Glucagon Assays}

For accurate quantification of glucagon levels in plasma samples by LC-HRMS [3], these plasma samples were subjected to protein precipitation followed by solid-phase extraction. Subsequently, extracted glucagon was measured using LC-HRMS. Plasma glucagon levels were measured by an LC-HRMS assay with parallel reaction monitoring (PRM) [3]. In brief, an automated nano-LCHRMS system was used that consisted of an Ultimate 3,000 Series nano-LC system and a Q Exactive Quadrupole-Orbitrap mass spectrometer (Thermo Fisher Scientific, GmbH, Bremen, Germany) equipped with a nanoelectron spray ionization interface and Black XYZ ion source (AMR, Inc., Tokyo, Japan). For accurate quantification of glucagon, 14-Leu-(13C6)-glucagon was added as an internal standard (IS) into every $200 \mu \mathrm{L}$ plasma sample. After precipitation of proteins, solid-phase extraction was performed to deplete plasma proteins and unnecessary peptides. PRM was performed by monitoring the sum of the peak areas of 5 fragment ions in the +4 -charge state for glucagon ${ }_{1-29}(\mathrm{~m} / z$ 871.66) and stable isotope-labeled-glucagon ${ }_{1-29}(\mathrm{~m} / z$ 873.17). To construct the calibration curve, the peak area ratios of glucagon to IS for each standard sample (ranging from 0.5 to $100 \mathrm{pmol} / \mathrm{L}$ ) and the known concentrations of the standard samples were plotted on the $y$ - and $x$-axes, respectively. Glucagon concentrations in plasma samples were calculated by linear regression with a $1 / \chi^{2}$ weighting factor. Plasma glucagon levels were also quantified using sandwich ELISA (Mercodia AB, Uppsala, Sweden), according to manufacturer's instructions. Moreover, the measurement of plasma glucagon levels with an ImmunoChem ${ }^{\mathrm{TM}}$ glucagon double antibody RIA kit (DIAsource ImmunoAssays S.A., Louvain-la-Neuve, Belgium) was performed at SRL, Inc. (Tokyo, Japan).

\section{Statistical Analysis}

If a measured glucagon level was below the limit of detection, half the lower limit was used in the statistical analysis for convenience. A paired $t$ test was used to compare G-LC/MS before and after a single HD session. The accuracy of plasma glucagon levels measured by sandwich ELISA (G-ELISA) and G-RIA was examined by comparison with G-LC/MS, which was used as the standard for glucagon levels in the present study. Pearson's correlation coefficient $(r)$ was calculated to assess the linear relationship between G-LC/MS and glucagon levels as measured by other assays. Then, Fisher's $z$-transformation was used to determine whether the 2 correlation coefficients were significantly different. In all statistical analyses, a 2-tailed $p<0.05$ threshold was considered statistically significant.

\section{Results}

The baseline patient characteristics and glucose concentrations in dialysates are shown in Table 1. From preHD levels, G-LC/MS was significantly decreased by 6.35 $\pm 5.65 \mathrm{pmol} / \mathrm{L}$ after HD $(p<0.001)$ (Fig. 1). G-ELISA was significantly correlated with G-LC/MS (overall $r=0.95$, $p<0.001$ ) irrespective of the timing of sampling (pre-HD $r=0.95, p<0.001$; post-HD $r=0.78, p<0.001$ ) (Fig. 2). Although G-RIA was significantly correlated with 
Table 1. Baseline clinical characteristics

\begin{tabular}{lc}
\hline$n$ & 26 \\
Age, years & $67.4 \pm 8.9$ \\
Male, $n(\%)$ & $20(76.9)$ \\
BMI, kg/m² & $23.5 \pm 3.2$ \\
HbA1c, \% & $6.4 \pm 0.9$ \\
GA, \% & $20.6 \pm 4.1$ \\
Duration of diabetes, years & $19.5 \pm 10.1$ \\
Duration of HD, years & $5.8 \pm 4.4$ \\
Primary disease of HD, $n(\%)$ & \\
$\quad$ Diabetic nephropathy & $21(80.8)$ \\
$\quad$ Other diseases & $5(19.2)$ \\
Glucose concentration in dialysate, $n(\%)$ & $12(46.2)$ \\
$\quad 100 \mathrm{mg} / \mathrm{dL}$ & $9(34.6)$ \\
$\quad 150 \mathrm{mg} / \mathrm{dL}$ & $5(19.2)$ \\
$125 \mathrm{mg} / \mathrm{dL}$ & \\
\hline
\end{tabular}

Date are presented as mean \pm standard deviation or median. $n$, number; HbA1c, glycated hemoglobin; GA, glycoalbumin; HD, hemodialysis.

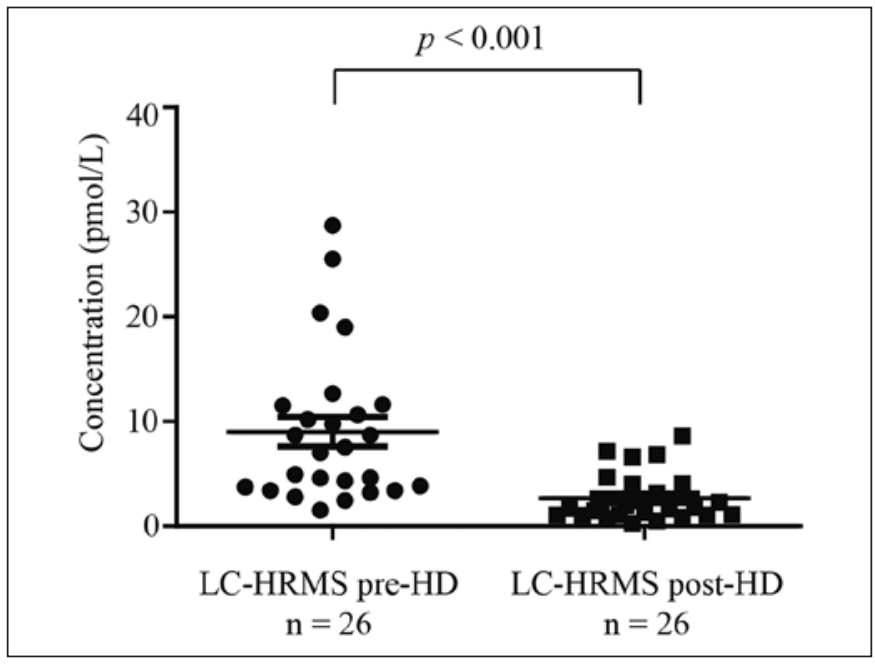

Fig. 1. Plasma glucagon levels measured by LC-HRMS. The mean difference in pre- and post-hemodialysis plasma glucagon was 6.35 $\pm 5.65 \mathrm{pmol} / \mathrm{L}(p<0.001, n=26)$. LC-HRMS, liquid chromatography-high-resolution mass spectrometry.

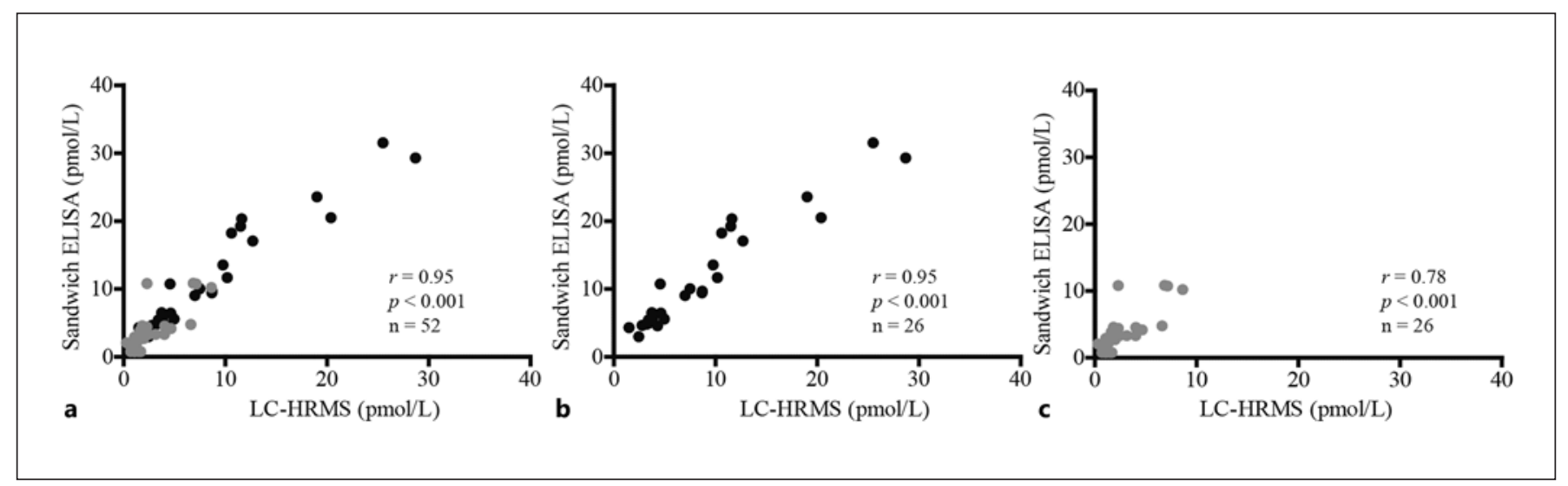

Fig. 2. Correlation between plasma glucagon levels measured by LC-HRMS and sandwich ELISA. a Correlation between plasma glucagon levels measured by LC-HRMS and sandwich ELISA before and after HD. Black and gray circles are pre- and post-HD values of plasma glucagon, respectively. Correlations of the plasma glucagon levels are shown separately for pre-HD (b) and post-HD (c) measurements. ELISA, enzyme-linked immunosorbent assay; HD, hemodialysis; LC-HRMS, liquid chromatography-high-resolution mass spectrometry.

G-LC/MS (overall $r=0.81, p<0.001$; pre-HD $r=0.81, p$ $<0.001$; post-HD $r=0.44, p=0.024$ ) (Fig. 3), overall $r$ and pre-HD $r$ were significantly lower than those between GELISA and G-LC/MS $(p<0.001$ and $p=0.011$, respectively) (Table 2). As shown in the scatter plots, G-RIA was generally much higher than G-LC/MS, whereas G-ELISA was closer to G-LC/MS. Mean blood glucose was significantly lower after HD $(132.2 \mathrm{mg} / \mathrm{dL})$ than before $\mathrm{HD}$ $(169.6 \mathrm{mg} / \mathrm{dL})(p<0.01)$.

\section{Discussion/Conclusion}

This was the first study in which glucagon levels were measured using LC-HRMS in HD patients with T2DM. Abe et al. [5] reported that G-RIA estimates were decreased during HD, and we showed that post-HD G-LC/ MS was much lower than pre-HD G-LC/MS. In addition, the glucagon levels decreased, even though the blood glucose levels decreased during the HD session. Although the details of the mechanism are still not 


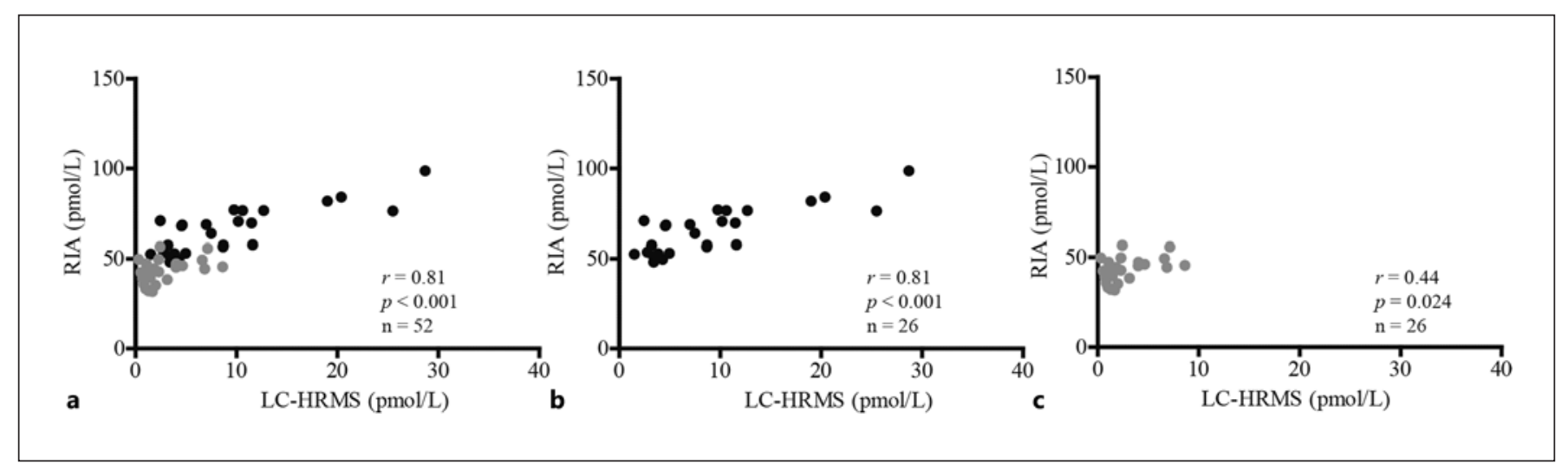

Fig. 3. Correlation between plasma glucagon levels measured by LC-HRMS and RIA before and after HD. a Correlation between plasma glucagon levels measured by LC-HRMS and RIA. Black and gray circles are pre- and post-HD values of plasma glucagon, respectively. Correlations of the plasma glucagon levels are shown separately for pre-HD (b) and post-HD (c) measurements. HD, hemodialysis; LC-HRMS, liquid chromatographyhigh-resolution mass spectrometry; RIA, radioimmunoassay.

known, one possible explanation is that glucagon and insulin might be removed by HD $[5,10]$. HD-related hypoglycemia is also a common problem in HD patients with or without T2DM [5]. Because investigating the details of glucagon dynamics during hemodialysis may help clarify this problem, further detailed studies are warranted.

Katahira et al. [1] reported that the accuracy of sandwich ELISA was equivalent to that of LC-HRMS for the analysis of glucagon in T2DM patients, but the accuracy of sandwich ELISA in HD patients with T2DM remains unclear. We also showed that sandwich ELISA could measure glucagon accurately in HD patients relative to RIA when our developed LC-HRMS method was used as a standard in this study. G-RIA was also significantly higher than G-LC/MS, as previously reported in a study of ESRD patients [11]. Thus, it was suggested that sandwich ELISA may provide effective glucagon measurements in HD patients. In a future study, we aim to investigate the details of glucagon levels, including changes due to different meals and medications, in HD patients and non-HD ESRD patients with or without T2DM.

This study had several limitations. First, all subjects control their T2DM with linagliptin, a DPP-4 inhibitor. Second, we did not investigate nondiabetic patients on maintenance HD. Third, we have not considered the effects of diet and physical activity. Because the subjects came to the hospital after breakfast in this study, postprandial blood samples were collected as the pre-HD samples. Moreover, they rested on the bed and did not
Table 2. Statistical analysis of correlations between each measurement method and LC-HRMS

\begin{tabular}{lccc}
\hline & \multicolumn{3}{l}{ Correlation coefficient $(r)$} \\
\cline { 2 - 4 } & $\begin{array}{l}\text { overall } \\
(n=52)\end{array}$ & $\begin{array}{l}\text { pre-HD } \\
(n=26)\end{array}$ & $\begin{array}{l}\text { post-HD } \\
(n=26)\end{array}$ \\
\hline G-ELISA and G-LC/MS & 0.950 & 0.954 & 0.778 \\
G-RIA and G-LC/MS & 0.814 & 0.807 & 0.441 \\
$p$ value & $<0.001$ & 0.011 & 0.054 \\
\hline
\end{tabular}

G-RIA was significantly correlated with G-LC/MS (overall $r=$ $0.814, p<0.001$; pre-HD $r=0.807, p<0.001$; post-HD $r=0.441$, $p=0.024)$; overall $r$ and pre-HD $r$ were significantly lower than those between G-ELISA and G-LC/MS $(p<0.001$ and $p=0.011$, respectively). G-ELISA, plasma glucagon levels measured by enzyme-linked immunosorbent assay; G-LC/MS, plasma glucagon levels measured by liquid chromatography-high-resolution mass spectrometry; G-RIA, plasma glucagon levels measured by radioimmunoassay; HD, hemodialysis.

have meals during HD sessions. Additional studies investigating glucagon dynamics are needed in hemodialysis patients with T2DM with more detailed consideration given to diet and physical activity.

In conclusion, this study was the first to measure glucagon levels using LC-HRMS in T2DM patients undergoing HD. Additionally, sandwich ELISA was able to accurately measure glucagon levels even in T2DM patients on maintenance HD. Additional studies investigating glucagon dynamics are needed to evaluate HD-related hypoglycemia in patients with T2DM. 


\section{Acknowledgments}

We thank Ms. Maiko Daisaka of Niigata University for providing technical assistance. We also thank ThinkSCIENCE Inc. (Tokyo, Japan) for English language editing.

\section{Statement of Ethics}

This study was approved by the institutional review boards of Niigata University (Approval No. 2015-2572) and Shinrakuen Hospital (Approval No. H28003) and was registered at the University Hospital Medical Information Network Clinical Trials Registry (UMIN-CTR; trial identification number: UMIN000024284) in Japan. All patients provided written informed consent for using their clinical data for research purposes.

\section{Conflict of Interest Statement}

M.H., H.K., T.K., I.N., and A.S. have received lecture fees from Eli Lilly Japan K.K., MSD K.K., and Nippon Boehringer Ingelheim Co., Ltd.; M.H. has received research support from Eli Lilly Japan
K.K. and MSD K.K.; I.N. and A.S. have received research support from Eli Lilly Japan K.K., MSD K.K., and Nippon Boehringer Ingelheim Co., Ltd.

\section{Funding Sources}

No funding or sponsorship was received for this study or publication of this article.

\section{Author Contributions}

Y.Y., M.H., and H.K. were responsible for the conception and design of the study. Y.Y. was the chief investigator and responsible for data analysis. M.H., H.K., and N.T. were responsible for data analysis. Y.Y., M.H., and H.K. were responsible for data acquisition. A.M., H.H, E.M., T.K., and M.K. were responsible for the glucagon measurements. Y.Y., M.H., H.K., N.T., A.M., H.H., E.M., T.K., M.K., I.N., and A.S. were responsible for data interpretation. Y.Y. and M.H. were responsible for drafting the manuscript. All authors contributed to the writing of the final manuscript.

\section{References}

1 Katahira T, Kanazawa A, Shinohara M, Koshibu M, Kaga H, Mita T, et al. Postprandial plasma glucagon kinetics in type 2 diabetes mellitus: comparison of immunoassay and mass spectrometry. J Endocr Soc. 2019;3(1): $42-51$

2 Kobayashi M, Satoh H, Matsuo T, Kusunoki Y, Tokushima M, Watada $\mathrm{H}$, et al. Plasma glucagon levels measured by sandwich ELISA are correlated with impaired glucose tolerance in type 2 diabetes. Endocr J. 2020;67(9): 903-22.

3 Miyachi A, Kobayashi M, Mieno E, Goto M, Furusawa K, Inagaki T, et al. Accurate analytical method for human plasma glucagon levels using liquid chromatography-high resolution mass spectrometry: comparison with commercially available immunoassays. Anal Bioanal Chem. 2017;409(25) 5911-8.
4 Kitamura $\mathrm{T}$, Watada $\mathrm{H}$, Nagasaka S, Ishihara $\mathrm{H}$, Shiba T, Ueki K, et al. Report of the Japan diabetes Society's committee on validation of glucagon assay methods: plasma glucagon levels measured by a novel system correlate with the pathology of diabetes. J Jpn Diabetes Soc. 2020;63(12):826-34

5 Abe M, Kalantar-Zadeh K. Haemodialysisinduced hypoglycaemia and glycaemic disarrays. Nat Rev Nephrol. 2015;11(5):302-13.

6 Jørgensen MB, Idorn T, Knop FK, Holst JJ, Hornum M, Feldt-Rasmussen B. Clearance of glucoregulatory peptide hormones during haemodialysis and haemodiafiltration in non-diabetic end-stage renal disease patients. Nephrol Dial Transplant. 2015;30(3):513-20.

7 Suzuki Y, Arakawa M. The treatment of the uraemic diabetic. Are we doing enough? A view from Japan. Fumitake Gejyo and collaborate study group. Nephrol Dial Transplant. 1995;10(Suppl 7):47-55.
8 Holst JJ, Christensen M, Lund A, de Heer J, Svendsen B, Kielgast U, et al. Regulation of glucagon secretion by incretins. Diabetes Obes Metab. 2011;13(Suppl 1):89-94.

9 Ichikawa R, Takano K, Fujimoto K, Motomiya T, Kobayashi M, Kitamura T, et al. Basal glucagon hypersecretion and response to oral glucose load in prediabetes and mild type 2 diabetes. Endocr J. 2019;66(8):663-75.

10 Abe M, Kaizu K, Matsumoto K. Plasma insulin is removed by hemodialysis: evaluation of the relation between plasma insulin and glucose by using a dialysate with or without glucose. Ther Apher Dial. 2007;11(4):280-7.

11 Wewer Albrechtsen NJ, Hartmann B, Veedfald S, Windeløv JA, Plamboeck A, BojsenMøller KN, et al. Hyperglucagonaemia analysed by glucagon sandwich ELISA: nonspecific interference or truly elevated levels? Diabetologia. 2014;57(9):1919-26. 